\title{
Immunomodulatory properties of Lactobacillus salivarius are not limited to the intestine
}

\author{
J. Galvez ${ }^{1}$, B. Arribas ${ }^{1}$, M. E. Rodríguez-Cabezas ${ }^{1}$, E. Bailon ${ }^{1}$, M. Comalada ${ }^{1}$, D. Camuesco ${ }^{1}$, \\ J. Xaus ${ }^{2}$ and A. Zarzuelo ${ }^{1}$ \\ ${ }^{1}$ Department of Pharmacology, University of Granada, Granada, Spain and ${ }^{2}$ Puleva Biotech, SA, Granada, Spain
}

\begin{abstract}
Previous studies have shown the beneficial effects exerted by probiotics on inflammatory bowel disease ${ }^{(1)}$, an intestinal condition characterized by an altered intestinal immune response ${ }^{(2)}$. However, it would be interesting to know whether the immunomodulatory properties of probiotics are restricted to a local effect in the intestine or whether their effect can also be extrapolated to other systemic immune alterations. The aim of the present study was to test the effect of a probiotic, Lactobacillus salivarius CECT5713, in two experimental models of local or systemic altered immune response, i.e. the trinitrobenzenesulfonic acid (TNBS) model of rat colitis and the lipopolysaccharide (LPS)-induced septic shock in mice. For this purpose, mice or rats $(n 10)$ were given the probiotic $\left(5 \times 10^{8}\right.$ colony-forming units/ml drinking water), starting 2 weeks before damage induction. A control group ( $n$ 10) without probiotic was also used for reference. Colitis was induced in rats by intracolonic administration of TNBS $(10 \mathrm{mg})$ and after 1 week was evaluated both histologically and biochemically (myeloperoxidase activity, glutathione content, inducible NO synthase (iNOS) expression) ${ }^{(3)}$. Septic shock was induced in mice by administering LPS $(40 \mathrm{mg} / \mathrm{kg}$, intraperitoneally) and the mice killed $24 \mathrm{~h}$ later, when colon and spleen were removed. Colonic iNOS expression was determined by Western blot, and activated T-cells were obtained from spleens by concanavalin A incubation and the immune response evaluated by RT-PCR or ELISA for different cytokines (IL-2, -5, -6 and -10). The results showed that L. salivarius was able to ameliorate both the local and systemic altered immune response. The probiotic exerted intestinal anti-inflammatory activity, since it significantly reduced the extension of the colonic damage induced by TNBS in comparison with non-treated colitic rats; this effect was accompanied by a $42 \%$ reduction in myeloperoxidase activity $(P<0.05)$, a $44 \%$ increase in glutathione content $(P<0.05)$ and a reduction in colonic iNOS. Moreover, the probiotic treatment significantly prevented the increase in colonic weight $(\mathrm{mg} / \mathrm{cm})$ induced by septic shock (264 (SE 15) v. 322 (SE 15); $P<0.05)$, without showing differences from normal mice (246 (SE 14)). Similarly, the LPS-induced colonic iNOS expression was lower in the probiotic-treated mice $(30 \%)$. LPS also stimulated the expression of different cytokines assayed in the splenocytes, while the probiotic-treated mice showed a reduction in cytokine expression of $80 \%$ for IL-5 and $100 \%$ for both IL-2 and IL-6 (Figure 1). IL-10 secretion was reduced in control mice $(603$ (sE 102) pg/ml; $P<0.05)$ in comparison with the normal group $(1064$ (SE 80$) \mathrm{pg} / \mathrm{ml})$, which was increased in probiotic-treated mice $(1034(\mathrm{SE} 150 \mathrm{pg} / \mathrm{ml} ; P<0.05)$ when compared with the LPS control group. In conclusion, the immunomodulatory properties of the probiotic $L$. salivarius are not restricted to the intestine, since it is also able to ameliorate the alteration in the systemic immune response derived from LPS administration to mice.
\end{abstract}

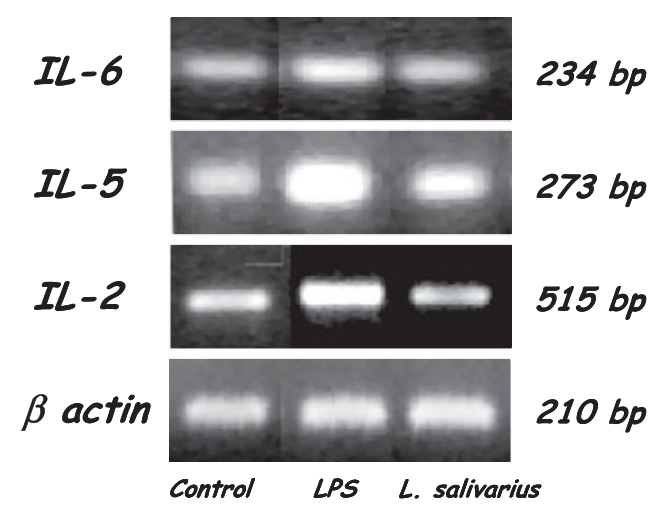

Figure 1. Lactobacillus salivarius administration reduced cytokine expression (RT-PCR) in LPS-induced septic shock in mice.

1. Ewaschuk JB \& Dieleman LA (2006) World J Gastroenterol 12, 5941-5950.

2. Hanauer SB (2006) Inflamm Bowel Dis 12, S3-S9.

3. Peran L, Sierra S, Comalada M et al. (2007) Br J Nutr 97, 96-103. 